\title{
Academic drug discovery within the United Kingdom: a reassessment
}

\author{
Emma Shanks, Robin Ketteler and Daniel Ebner
}

In 2014, Tralau-Stewart et al. published an overview of academic drug discovery efforts in the United Kingdom based on a survey they conducted (UK academic drug discovery. Nature Rev. Drug Discov. 13, 15-16 $(2014))^{1}$. They observed that academic screening within the United Kingdom is comparable to that in the United States (discussed in REF. 2) with regard to primary therapeutic focus (with cancer, infectious disease and cardiovascular disease constituting the most highly prioritized therapeutic indications) and areas of unmet medical need. Parallels were also drawn between the motivational drivers and annual operating costs of screening in both countries. However, regarding infrastructure, it was reported that most drug discovery programmes in academic screening groups (ASGs) in the United Kingdom were conducted in a traditional research group (that is, a team of postdoctoral researchers, Ph.D. students and technicians led by a single principal investigator), with only $13 \%$ of groups operating in centres dedicated to drug discovery. The most surprising finding was that "access to high-throughput screening (HTS) facilities and associated compound libraries were not reported by any UK group" (REF. 1). Tralau-Stewart et al. clearly stated that the responses provided a "snapshot" of academic research at the time of the survey (2013) and not a comprehensive analysis, as some groups may not have received or responded to the survey ${ }^{1}$. However, it seems to us that the presence of centre-led ASGs and industry-standard drug discovery programmes conducted within an academic environment in the United Kingdom was substantially understated by the survey results. Therefore, we conducted another investigation of the academic drug discovery landscape in the United Kingdom by identifying academic drug discovery units and facilities and analysing their characteristics, which we discuss here.

\section{UK drug discovery facilities}

We identified 24 dedicated drug discovery facilities in the United Kingdom (TABLE 1; see also the UK Drug Discovery Consortium website in Further information). These facilities have a range of operational frameworks: some are academic facilities operated by universities with several sources of funding; several are operated by charitable organizations; and others are industrial facilities sited on or near to academic campuses. The common theme linking these groups is that they are all open-access drug discovery facilities that are accessible by UK-wide academic groups, and each facility employs some personnel from academia, thereby strengthening links to the UK academic community. These groups may therefore be better referred to collectively as academic and not-for-profit screening groups; hereon, we refer to them as ASGs.

The scale of the undertaking and resourcing of these groups varies according to the objectives of each facility. Some groups provide dedicated support for distinct aspects of the drug discovery pipeline (for example, target discovery or identification of chemical starting points for nominated disease indications) and are resourced with the relevant expertise and technical knowledge. At least six ASGs conduct industry-standard drug discovery, resourced largely by professionals with substantial industrial experience, and they incorporate extensive in-house medicinal chemistry expertise and drug metabolism and pharmacokinetics (DMPK) resources.

All of the identified groups reported that they had conducted multiple highthroughput screens (HTSs) in the past year (TABLE 1). They actively pursue primary screen hits for further validation or drug development, and they regularly publish their results in peer-reviewed scientific journals. Additionally, we found that all of the groups use industry-standard liquid handling and/or readout instruments and have research staff dedicated to each step of the drug discovery pipeline (for example, compound management, assay development, screening and data analysis) suggesting that their facilities are well equipped with both instrumentation and personnel. These facilities represent a major investment in UK drug discovery research. The similarity in facility numbers between the United Kingdom and the United States highlights a clear commitment in the United Kingdom to provide the infrastructure necessary to support academic screening.

\section{Funding and collaboration}

Currently, the majority of funding for the main UK-based ASGs is provided by the UK government, with considerable support coming from universities, UK-based and international charitable organizations (for example, Cancer Research UK (CRUK), which supports programmes at 5 of the 23 facilities), and the UK Medical Research Council. In addition to government and charitable funding streams, we have recently experienced a paradigm shift in the perception of academic screening by the industrial sector. Pharmaceutical companies now recognize the potential of academic alliances and several have entered into partnerships with UK-based ASGs. Collaborative efforts to address a broad range of disease areas are underway (TABLE 1), through the provision of well-characterized compound collections (GlaxoSmithKline (GSK) and Pfizer) and engaging closely with academic partners to enhance their own drug discovery capacity. Notable examples include AstraZeneca's Open Innovation Initiative, GSK's Centre for Therapeutic Target Validation (CTTV) and the Eisai-University College London (UCL) collaborative drug discovery alliance. These partnerships are evidence that the UK academic screening community is held in high esteem by global pharmaceutical companies.

\section{Screening platforms and technologies} The approaches used across ASGs are multidisciplinary yet complementary: the identification and de-risking of potential targets using high-throughput functional genomics helps to bridge the gap between the academic research and drug discovery disciplines ${ }^{3,4}$. Platforms such as RNA-mediated interference (RNAi) screening can support target identification and concurrently provide novel biological insights. Moreover, dedicated efforts to unearth novel chemical starting points for drug discovery are increasing, supplemented by committed medicinal and/or computational chemistry for the development of lead compounds. The repurposing of existing therapeutics is increasingly complementing the more traditional drug discovery workflow as more groups begin to use screening to explore the potential applications of existing drugs for alternative indications ${ }^{5}$.

ASGs have also been a driving force behind the development and implementation of highcontent imaging and analysis in drug discovery. We found that all of the UK-based ASGs that are engaged in cell-based phenotypic screening use state-of-the-art high-content imagers, and most use multiple instruments. Other platforms typically perceived to be restricted to use only by pharmaceutical 


\begin{tabular}{|c|c|c|c|c|c|c|c|c|}
\hline Facility & $\begin{array}{l}\text { Host } \\
\text { institution }\end{array}$ & $\begin{array}{l}\text { Main disease } \\
\text { indications }\end{array}$ & $\begin{array}{l}\text { Funding } \\
\text { sources }\end{array}$ & Staff & Platforms & Technologies & $\begin{array}{l}\text { No. of } \\
\text { screens } \\
\text { per } \\
\text { year }\end{array}$ & $\begin{array}{l}\text { Medicinal } \\
\text { chemistry } \\
\text { follow-up }\end{array}$ \\
\hline $\begin{array}{l}\text { Dundee Drug } \\
\text { Discovery } \\
\text { Unit }^{\ddagger}\end{array}$ & $\begin{array}{l}\text { University of } \\
\text { Dundee }\end{array}$ & $\begin{array}{l}\text { Diseases of the } \\
\text { developing world } \\
\text { and innovative } \\
\text { targets and pathways }\end{array}$ & $\begin{array}{l}\text { UK government, } \\
\text { charities and } \\
\text { industry }\end{array}$ & $>70$ & $\begin{array}{l}\text { Biophysical, } \\
\text { biochemical and } \\
\text { cellular assays }\end{array}$ & Multiple & NA & Yes \\
\hline $\begin{array}{l}\text { National } \\
\text { Phenotypic } \\
\text { Screening } \\
\text { Centre }\end{array}$ & $\begin{array}{l}\text { University } \\
\text { of Dundee, } \\
\text { University of } \\
\text { Oxford and } \\
\text { University of } \\
\text { Edinburgh }\end{array}$ & $\begin{array}{l}\text { All diseases, with } \\
\text { human prioritized } \\
\text { over non-human }\end{array}$ & $\begin{array}{l}\text { UK government, } \\
\text { charities and } \\
\text { industry }\end{array}$ & $\sim 25$ & $\begin{array}{l}\text { Small molecules, } \\
3 \mathrm{D} \text {, tissue, patient } \\
\text { cells, iPS cells } \\
\text { and genome } \\
\text { engineering }\end{array}$ & $\begin{array}{l}\mathrm{HCl}, \mathrm{MPA}, \\
\text { label-free and } \mathrm{HT} \\
\text { flow cytometry }\end{array}$ & $10-15$ & Yes \\
\hline $\begin{array}{l}\text { European } \\
\text { Screening } \\
\text { Centre } \\
\text { Newhouse } \\
\text { and European } \\
\text { Lead Factory }\end{array}$ & $\begin{array}{l}\text { Multiple } \\
\text { sites across } \\
\text { the United } \\
\text { Kingdom } \\
\text { (including } \\
\text { Dundee and } \\
\text { Lanarkshire) } \\
\text { and Europe }\end{array}$ & All human diseases & $\begin{array}{l}\text { Innovative } \\
\text { Medicines } \\
\text { Initiative } \\
\text { and in-kind } \\
\text { contributions } \\
\text { from EFPIA } \\
\text { participants }\end{array}$ & 50 & $\begin{array}{l}\text { Small molecules } \\
\left(326,000^{\S}\right) \text {, } \\
\text { biophysical and } \\
\text { biochemical } \\
\text { assays }\end{array}$ & $\begin{array}{l}\text { Ultra-HTS } \\
\text { plate-reader, SPR } \\
\text { and label-free }\end{array}$ & 30 & Yes \\
\hline $\begin{array}{l}\text { Edinburgh } \\
\text { Cancer } \\
\text { Discovery } \\
\text { Unit }\end{array}$ & $\begin{array}{l}\text { Edinburgh } \\
\text { Cancer } \\
\text { Research } \\
\text { Centre, } \\
\text { University of } \\
\text { Edinburgh }\end{array}$ & Oncology & $\begin{array}{l}\text { Industry } \\
\text { alliances, MRC } \\
\text { and University } \\
\text { of Edinburgh }\end{array}$ & 14 & $\begin{array}{l}\text { Small molecules } \\
\text { and chemical } \\
\text { library synthesis }\end{array}$ & $\begin{array}{l}\mathrm{HCl}, \mathrm{MPA} \text {, image } \\
\text { informatics, } \\
\text { reverse phase } \\
\text { protein array and } \\
\text { dual ligand }\end{array}$ & 12 & Yes \\
\hline $\begin{array}{l}\text { Beatson } \\
\text { Institute RNAi } \\
\text { Screening } \\
\text { Facility }\end{array}$ & $\begin{array}{l}\text { CRUK Beatson } \\
\text { Institute, } \\
\text { University } \\
\text { of Glasgow }\end{array}$ & Oncology & CRUK & 5 & $\begin{array}{l}\text { Genome- } \\
\text { wide human and } \\
\text { mouse RNAi and } \\
\text { drug repurposing }\end{array}$ & $\begin{array}{l}\mathrm{HCl}, \mathrm{MPA} \text { and } \\
\mathrm{HTS} \text { plate reader }\end{array}$ & 8 & No \\
\hline $\begin{array}{l}\text { Queen's } \\
\text { University } \\
\text { Belfast Drug } \\
\text { Discovery } \\
\text { Group }\end{array}$ & $\begin{array}{l}\text { Queen's } \\
\text { University, } \\
\text { Belfast }\end{array}$ & Oncology & $\begin{array}{l}\text { Almac, Queen's } \\
\text { University and } \\
\text { Invest Northern } \\
\text { Ireland }\end{array}$ & 20 & $\begin{array}{l}\text { Fragments, } \\
\text { CADD and virtual } \\
\text { screening }\end{array}$ & $\begin{array}{l}\text { SPR, } \\
\text { thermophoresis, } \\
\text { Schrodinger, } \\
\text { MOE, HCl } \\
\text { and MPA }\end{array}$ & 3 & Yes \\
\hline $\begin{array}{l}\text { Medicinal } \\
\text { Chemistry } \\
\text { and Chemical } \\
\text { Biology } \\
\text { Technology } \\
\text { Group }\end{array}$ & $\begin{array}{l}\text { University } \\
\text { of Leeds }\end{array}$ & All human diseases & $\begin{array}{l}\text { University } \\
\text { of Leeds, UK } \\
\text { government, } \\
\text { charities and } \\
\text { industry }\end{array}$ & 5 & $\begin{array}{l}\text { Small molecules } \\
(50,000), \text { drug } \\
\text { repurposing, } \\
\text { fragments and } \\
\text { virtual screening }\end{array}$ & $\begin{array}{l}\text { Biochemical, } \\
\text { phenotypic and } \\
\text { biophysical }\end{array}$ & $5-10$ & Yes \\
\hline $\begin{array}{l}\text { Bioscreening } \\
\text { Technology } \\
\text { Group }\end{array}$ & $\begin{array}{l}\text { University } \\
\text { of Leeds }\end{array}$ & All human diseases & $\begin{array}{l}\text { University } \\
\text { of Leeds, UK } \\
\text { government, } \\
\text { charities and } \\
\text { European } \\
\text { Commission }\end{array}$ & 2 & $\begin{array}{l}\text { Genome- } \\
\text { wide human and } \\
\text { mouse RNAi, } \\
\text { miRNA inhibitors } \\
\text { and mimics, } \\
\text { small-molecule } \\
\text { screening and } \\
\text { drug repurposing }\end{array}$ & $\begin{array}{l}\mathrm{HCl}, \mathrm{MPA} \text { and } \\
\mathrm{HTS} \text { plate reader }\end{array}$ & $5-8$ & Yes \\
\hline $\begin{array}{l}\text { Adhiron } \\
\text { Screening } \\
\text { Facility }\end{array}$ & $\begin{array}{l}\text { University } \\
\text { of Leeds }\end{array}$ & All human diseases & $\begin{array}{l}\text { University } \\
\text { of Leeds, UK } \\
\text { government, } \\
\text { charities and } \\
\text { industry }\end{array}$ & 19 & Biologics & $\begin{array}{l}\text { Phage display, } \\
\text { biochemical and } \\
\text { biophysical }\end{array}$ & 150 & Yes \\
\hline
\end{tabular}




\begin{tabular}{|c|c|c|c|c|c|c|c|c|}
\hline Facility & $\begin{array}{l}\text { Host } \\
\text { institution }\end{array}$ & $\begin{array}{l}\text { Main disease } \\
\text { indications }\end{array}$ & $\begin{array}{l}\text { Funding } \\
\text { sources }\end{array}$ & Staff & Platforms & Technologies & $\begin{array}{l}\text { No. of } \\
\text { screens } \\
\text { per } \\
\text { year }\end{array}$ & $\begin{array}{l}\text { Medicinal } \\
\text { chemistry } \\
\text { follow-up }\end{array}$ \\
\hline $\begin{array}{l}\text { Manchester } \\
\text { Institute Drug } \\
\text { Discovery } \\
\text { Unit }\end{array}$ & $\begin{array}{l}\text { CRUK } \\
\text { Manchester } \\
\text { Institute, } \\
\text { University of } \\
\text { Manchester }\end{array}$ & Oncology & CRUK & 5 & $\begin{array}{l}\text { Small molecules } \\
\text { and drug } \\
\text { repurposing }\end{array}$ & $\mathrm{HCl}$ & $5-10$ & Yes \\
\hline $\begin{array}{l}\text { Sheffield RNAi } \\
\text { Screening } \\
\text { Facility }\end{array}$ & $\begin{array}{l}\text { University of } \\
\text { Sheffield }\end{array}$ & $\begin{array}{l}\text { Oncology, Parkinson } \\
\text { disease and } \\
\text { myeloproliferative } \\
\text { neoplasms }\end{array}$ & $\begin{array}{l}\text { Wellcome } \\
\text { Trust, Yorkshire } \\
\text { Cancer } \\
\text { Research and } \\
\text { University of } \\
\text { Sheffield }\end{array}$ & 2 & $\begin{array}{l}\text { Genome-wide } \\
\text { drosophila RNAi, } \\
\text { human siRNA and } \\
\text { small molecules }\end{array}$ & $\begin{array}{l}\mathrm{HCl}, \mathrm{MPA} \text { and } \\
\text { HTS plate reader }\end{array}$ & 16 & No \\
\hline $\begin{array}{l}\text { SITraN Drug } \\
\text { Discovery } \\
\text { Suite }\end{array}$ & $\begin{array}{l}\text { University of } \\
\text { Sheffield }\end{array}$ & $\begin{array}{l}\text { Motor neuron } \\
\text { disease and } \\
\text { Parkinson disease }\end{array}$ & $\begin{array}{l}\text { Wellcome Trust, } \\
\text { MRCT, MNDA } \\
\text { and Parkinson's } \\
\text { UK }\end{array}$ & 6 & Small molecules & $\begin{array}{l}\mathrm{HCl}, \mathrm{MPA}, \mathrm{HTS} \\
\text { plate reader } \\
\text { and metabolic } \\
\text { outputs }\end{array}$ & 2 & No \\
\hline $\begin{array}{l}\text { Zebrafish } \\
\text { Small } \\
\text { Molecule } \\
\text { Screening } \\
\text { Unit }\end{array}$ & $\begin{array}{l}\text { University of } \\
\text { Sheffield }\end{array}$ & $\begin{array}{l}\text { Zebrafish models } \\
\text { of disease, } \\
\text { including epilepsy, } \\
\text { inflammation, } \\
\text { infection, hypoxia } \\
\text { and ototoxicity }\end{array}$ & $\begin{array}{l}\text { MRC, BBSRC, } \\
\text { charities and } \\
\text { industry }\end{array}$ & 1 & Small molecules & $\begin{array}{l}\mathrm{HCl}, \mathrm{MPA}, \mathrm{HTS} \\
\text { plate reader and } \\
\text { microscopy }\end{array}$ & $5-10$ & Yes \\
\hline $\begin{array}{l}\text { Drug } \\
\text { Discovery } \\
\text { Priority Group }\end{array}$ & $\begin{array}{l}\text { University of } \\
\text { Nottingham }\end{array}$ & All human diseases & $\begin{array}{l}\text { Charities, } \\
\text { industry and } \\
\text { the European } \\
\text { Union }\end{array}$ & 2 & Small molecules & $\begin{array}{l}\mathrm{HCl} \text { and } \mathrm{HTS} \\
\text { plate reader }\end{array}$ & $2-4$ & Yes \\
\hline $\begin{array}{l}\text { Birmingham } \\
\text { Drug } \\
\text { Discovery } \\
\text { Facility }\end{array}$ & $\begin{array}{l}\text { University of } \\
\text { Birmingham }\end{array}$ & $\begin{array}{l}\text { Infectious diseases } \\
\text { (tuberculosis, and } \\
\text { Salmonella enterica } \\
\text { and Staphylococcus } \\
\text { aureus infection), } \\
\text { leukaemia, lym- } \\
\text { phoma and HBV }\end{array}$ & $\begin{array}{l}\text { MRC, Wellcome } \\
\text { Trust and ERC }\end{array}$ & 3 & Small molecules & HTS plate reader & 5 & Yes \\
\hline $\begin{array}{l}\text { CRT Discovery } \\
\text { Laboratory }\end{array}$ & $\begin{array}{l}\text { Babraham } \\
\text { Research } \\
\text { Campus, } \\
\text { Cambridge }\end{array}$ & Oncology & $\begin{array}{l}\text { Industry } \\
\text { alliances, CRT } \\
\text { and CRUK }\end{array}$ & 74 & $\begin{array}{l}\text { Small molecules } \\
(>100,000) \text {, } \\
\text { fragments and } \\
\text { RNAi }\end{array}$ & $\begin{array}{l}\mathrm{HCI}, \mathrm{MPA}, \mathrm{HTS} \\
\text { plate reader, } \\
\text { MSD technology, } \\
\text { SPR, ITC and } \\
\text { crystallography }\end{array}$ & 6 & Yes \\
\hline $\begin{array}{l}\text { Target } \\
\text { Discovery } \\
\text { Institute }\end{array}$ & $\begin{array}{l}\text { University of } \\
\text { Oxford }\end{array}$ & $\begin{array}{l}\text { Oncology, } \\
\text { cardiovascular } \\
\text { and neuro- } \\
\text { degeneration }\end{array}$ & $\begin{array}{l}\text { UK government, } \\
\text { University } \\
\text { of Oxford, } \\
\text { UK charities, } \\
\text { industry and } \\
\text { SRFs }\end{array}$ & $>50$ & $\begin{array}{l}\text { Small molecules } \\
(50,000), \text { drug } \\
\text { repurposing, } \\
\text { fragments and } \\
\text { RNAi }\end{array}$ & $\begin{array}{l}\mathrm{HCl}, \mathrm{HTS} \text { plate } \\
\text { reader, RT-PCR } \\
\text { and HTFACS }\end{array}$ & 20 & Yes \\
\hline $\begin{array}{l}\text { CRUK Cancer } \\
\text { Therapeutics } \\
\text { Unit }\end{array}$ & $\begin{array}{l}\text { The Institute } \\
\text { of Cancer } \\
\text { Research, } \\
\text { London }\end{array}$ & Oncology & $\begin{array}{l}\text { CRUK, ICR, } \\
\text { Wellcome Trust } \\
\text { and industrial } \\
\text { collaborators }\end{array}$ & 143 & $\begin{array}{l}\text { Small molecules } \\
(210,000), \\
\text { fragments }(2,000) \\
\text { and siRNA }\end{array}$ & $\begin{array}{l}\mathrm{HCI}, \mathrm{HTS} \text { plate } \\
\text { reader, mobility } \\
\text { shift assays, SPR, } \\
\text { ITC, DSF and } \\
\text { crystallography }\end{array}$ & 3 & Yes \\
\hline $\begin{array}{l}\text { High- } \\
\text { throughput } \\
\text { Screening } \\
\text { Facility }\end{array}$ & Crick Institute & Oncology & CRUK & 4 & $\begin{array}{l}\text { Genome-wide } \\
\text { RNAi, small } \\
\text { molecules }(4,000) \\
\text { and fragments }\end{array}$ & $\begin{array}{l}\mathrm{HCl}, \mathrm{MPA}, \mathrm{HTS} \\
\text { plate reader, } \\
\text { thermal melt and } \\
\text { FACS }\end{array}$ & $20-30$ & No \\
\hline $\begin{array}{l}\text { Laboratory } \\
\text { for Molecular } \\
\text { Cell Biology }\end{array}$ & $\begin{array}{l}\text { University } \\
\text { College } \\
\text { London }\end{array}$ & $\begin{array}{l}\text { Neurodegeneration, } \\
\text { oncology, HIV, cell } \\
\text { biology }\end{array}$ & MRC & 4 & $\begin{array}{l}\text { RNAi, cDNA, } \\
\text { small molecules, } \\
\text { CRISPR }\end{array}$ & $\begin{array}{l}\mathrm{HCl}, \mathrm{MPA}, \mathrm{HTS} \\
\text { plate reader }\end{array}$ & $15-20$ & Yes \\
\hline MRCT & MRC, London & $\begin{array}{l}\text { All including } \\
\text { oncology, fibrosis, } \\
\text { neurodegeneration, } \\
\text { inflammation, } \\
\text { anti-infectives, pain, } \\
\text { autoimmune disease, } \\
\text { Cushing disease }\end{array}$ & $\begin{array}{l}\text { Self-funded } \\
\text { through } \\
\text { income from } \\
\text { translational } \\
\text { activities }\end{array}$ & & $\begin{array}{l}\text { Small molecules } \\
(>250,000) \text {, phage } \\
\text { and antibodies }\end{array}$ & $\begin{array}{l}\mathrm{HCl}, \mathrm{MPA}, \mathrm{HTS} \\
\text { plate reader, label } \\
\text { free, SPR, ITC, } \\
\text { thermal shift }\end{array}$ & $8-10$ & Yes \\
\hline
\end{tabular}




\begin{tabular}{|c|c|c|c|c|c|c|c|c|}
\hline Facility & $\begin{array}{l}\text { Host } \\
\text { institution }\end{array}$ & $\begin{array}{l}\text { Main disease } \\
\text { indications }\end{array}$ & $\begin{array}{l}\text { Funding } \\
\text { sources }\end{array}$ & Staff & Platforms & Technologies & $\begin{array}{l}\text { No. of } \\
\text { screens } \\
\text { per } \\
\text { year }\end{array}$ & $\begin{array}{l}\text { Medicinal } \\
\text { chemistry } \\
\text { follow-up }\end{array}$ \\
\hline $\begin{array}{l}\text { Translational } \\
\text { Drug } \\
\text { Discovery } \\
\text { Group }\end{array}$ & $\begin{array}{l}\text { University of } \\
\text { Sussex }\end{array}$ & $\begin{array}{l}\text { Oncology, } \\
\text { neuroscience }\end{array}$ & $\begin{array}{l}\text { Wellcome Trust, } \\
\text { CRUK }\end{array}$ & 18 & $\begin{array}{l}\text { Structure-based } \\
\text { drug discovery }\end{array}$ & $\begin{array}{l}\text { Biophysical, } \\
\text { crystallography, } \\
\text { virtual screening, } \\
\text { high-throughput } \\
\text { electrophysiology }\end{array}$ & 4 & Yes \\
\hline
\end{tabular}

ARUK, Alzheimer's Research UK; BBSRC, Biotechnology and Biological Sciences Research Council; BHF, British Heart Foundation; CADD, combined annotation dependent depletion; CRISPR, clustered regularly interspaced short palindromic repeats; CRT, Cancer Research Technology; CRUK, Cancer Research UK; DSF, differential scanning fluorimetry; EFPIA, European Federation of Pharmaceutical Industries and Associations; ERC, European Research Council; FACS, fluorescence-activated cell sorting; HBV, hepatitis B virus; $\mathrm{HCl}$, high-content imaging; HT, high-throughput; HTS, HT screening; iPS cells, induced pluripotent stem cells; ICR, Institute of Cancer Research; ITC, isothermal titration calorimetry; miRNA, microRNA; MNDA, Motor Neurone Disease Association; MOE, multivariate optical element; MPA, multiparametric phenotypic analysis; MRC, Medical Research Council; MRCT, MRC Technology; MSD, mesoscale delivery; NA, not applicable (information unavailable); NMR, nuclear magnetic resonance; RNAi, RNA-mediated interference; RT-PCR, real-time PCR; siRNA, short interfering RNA; SITraN, Sheffield Institute for Translational Neuroscience; SPR, surface plasmon resonance; SRFs, small research facilities; SULSA, Scottish Universities Life Science Alliance. ${ }^{*}$ The authors apologize to any academic screening group (ASG) they may have overlooked. ASGs are ordered from north to south within the UK. ${ }^{*}$ Data collated from facility website. ${ }^{\S}$ To be expanded to 500,000 compounds by 2017 .

companies, such as acoustic dispensing, are now well embedded in ASGs, with a total of 19 Echo systems (Labcyte) at 14 ASG sites.

Tralau-Stewart et al. also reported an apparent lack of uptake of HTS (defined as screening collections of $>100,000$ compounds) in UK-based ASGs. However, at the time of their survey, both the CRUK Cancer Therapeutics Unit at the Institute of Cancer Research and the Cancer Research Technology (CRT) Discovery Laboratory were regularly screening $>100,000$ compounds. Along with the Medical Research Council Technology (MRCT) and Dundee Drug Discovery Unit, these centres are capable of large-scale screening, although it is still not commonplace in ASGs. Given the limitations in funding, this will probably remain the case, as it is, arguably, unnecessary to duplicate such efforts. Typically, ASGs use either high-diversity libraries of 10,000-50,000 compounds or smaller, more focused compound sets. Notably, there has recently been a dramatic change in the perception of the necessity of screening very large libraries of compounds to identify the most appropriate starting points for innovative drug discovery, catalysed by understanding of the the substantial contribution of phenotypic approaches in identifying first-in-class drugs ${ }^{6}$. The focus has shifted to screening fewer, more discrete, welldesigned and diverse compound libraries. When coupled with advanced computational approaches to explore chemical space in silico ${ }^{7}$, it becomes evident that the size of screening collections is not necessarily the most crucial criterion for success in primary HTS.

\section{Discussion}

Screening within an academic environment forms an integral part of drug discovery initiatives within the United Kingdom, and efforts in this area have undergone dramatic expansion over the past 10 years. Furthermore, the strategic alliance between academia, the UK government and the pharmaceutical industry is exemplified by the $£ 8$ million investment into the National Phenotypic Screening Centre (NPSC, 2014; sourced principally from the Scottish Government via the Scottish Funding Council with both academia and industry supporting running costs) and by the establishment of the European Screening Centre (which supports researchers and small- and medium-sized enterprises in screening $~ 300,000$ compounds, collectively contributed by 7 pharmaceutical companies). While the sources and timescales of funding continue to evolve, the level of investment in the infrastructure and research of UK-based ASGs may represent an emerging cultural shift in the recognition of the value of academic drug discovery efforts.

Emma Shanks is at the Cancer Research UK Beatson Institute, Glasgow G61 1BD, UK

Robin Ketteler is at the Translational Research Resource Center, Medical Research Council Laboratory for Molecular Cell Biology, University College London, London WC1E 6BT, UK.

Daniel Ebner is at the Target Discovery Institute, Oxford OX3 7FZ, UK. Correspondence to E.S. e-mail:e.shanks@beatson.gla.ac.uk doi:10.1038/nrd4661

Tralau-Stewart, C., Low, C. M. R. \& Marlin, N. UK academic drug discovery. Nat. Rev. Drug Discov. 13, 15-16 (2014).

2. Frye, S. et al. US academic drug discovery. Nat. Rev, Drug Discov. 10, 409-410 (2011).

3. Shanks, E. J. Strategic siRNA screening approaches to target cancer at the Cancer Research UK Beatson Institute. Comb. Chem. High Throughput Screen 17, 328-332 (2014).

4. Brown, S. The Sheffield RNAi Screening Facility (SRSF): portfolio growth and technology development. Comb. Chem. High Throughput Screen 17, 319-321 (2014).

5. Shanks, E. Reduce, reuse, recycle: how drug repositioning is finding its niche in drug discovery. Eur. Pharm. Rev. 18, 31-34 (2013).

6. Swinney, D. C. $\&$ Anthony, J. How were new medicines discovered? Nat. Rev. Drug Discov. 10, 507-519 (2011).
7. Kar, S. \& Roy, K. How far can virtual screening take us in drug discovery? Expert Opin. Drug Discov. 8, 245-261 (2013).

Competing interests statement

The authors declare no competing interests.

FURTHER INFORMATION

Adhiron Screening Facility: http://www.fbs.leeds.ac.uk/staff/ profile.php?tag=Tomlinson D

Beatson Institute Drug Discovery Unit: http://www.beatson. gla.ac.uk/drug-discovery/drug-discovery.html Beatson Institute RNAi Screening Facility:

http://www.beatson.gla.ac.uk/advanced-technologies/emmashanks-rnai-screening.html

Birmingham Drug Discovery Facility:

http://www.birmingham.ac.uk/facilities/bddf/index.aspx Bioscreening Technology Group: http://medhealth.leeds. ac.uk/info/360/facilities/909/bioscreening technology group CRT Discovery Laboratory:

http://www.cancertechnology.co.uk/

CRUK Cancer Therapeutics Unit: http://www.icr.ac.uk/ourresearch/our-research-centres/cancer-research-uk-cancertherapeutics-unit

Drug Discovery Priority Group: http://nottingham.ac.uk/

research/priorities/drugdiscovery/index.aspx

Dundee Drug Discovery Unit:

http://www.drugdiscovery.dundee.ac.uk/home

Edinburgh Cancer Discovery Unit:

http://www.ecrc.ed.ac.uk/discovery-unit/overview.html

European Lead Factory: http://www.europeanleadfactory.eu European Screening Centre Newhouse:

http://www.lifesci.dundee.ac.uk/research/esc

High-throughput Screening Facility: http://www.crick.ac.uk/ Laboratory for Molecular Cell Biology: http://www.ucl.ac.uk/ Imcb/about-translational-research-resource-center

Manchester Institute Drug Discovery Unit:

http://www.cruk.manchester.ac.uk/Research/CRUK-MI-

Groups/Drug-Discovery/Home

Medicinal Chemistry and Chemical Biology Technology Group: http://medhealth.leeds.ac.uk/info/1930/medicinal chemistry and chemical biology technology group MRCT: http://www.mrctechnology.org/about-us/ Queen's University Belfast Drug Discovery group: http://www.qub.ac.uk/research-centres/CentreforCancer ResearchCellBiology/Research/EnablingTechnologies/ DrugDiscovery

National Phenotypic Screening Centre:

http://www.sulsa.ac.uk/research-facilities/uk-nationalphenotypic-screening-centre

Scottish Bioscreening Facility: http://www.gla.ac.uk/ researchinstitutes/iii/facilities/imaging/sbfglasgow/ Sheffield RNAi Screening Facility: http://www.rnai.group.shef.ac.uk SITraN Drug Discovery Suite: $\underline{\text { http://sitran.dept.shef.ac.uk/ }}$ Target Discovery Institute: http://www.tdi.ox.ac.uk/home Translational Discovery Group:

http:// www.sussex.ac.uk/lifesci/drugdiscovery/

UK Drug Discovery Consortium: http://www.ukddc.org/ Zebrafish Small Molecule Screening Unit:

http://www.bateson.group.shef.ac.uk/research/facilities/ sheffield-zebrafish-screening-facility/

ALL LINKS ARE ACTIVE IN THE ONLINE PDF 